\title{
Lesión de Dieulafoy rectal: una causa rara, pero potencialmente mortal de hemorragia del tubo digestivo bajo
}

\author{
Dieulafoy's rectal injury: A rare but potentially \\ fatal cause of lower GI bleeding
}

\author{
Benjamín Gallo Arriaga, ${ }^{*}{ }^{\ddagger}$ José Raúl Nieto Saucedo, ${ }^{\ddagger}$ Benjamín Gallo Chico, ${ }^{*}$ \\ J Jesús Ibarra Rodríguez, ${ }^{*}$ Karla Edith Santibáñez Bedolla, ${ }^{\ddagger}$ Carlos Hidalgo Valadez,,‡
}

\section{Resumen}

La hemorragia gastrointestinal es un cuadro frecuente, tanto en consulta externa como en la Sala de Urgencias, cuya etiología puede ser diversa. La lesión de Dieulafoy, descrita por primera vez en 1898, es una de las causas más rara, pero potencialmente mortal por su dificultad diagnóstica. Se presenta el caso hombre de 81 años, quien ingresó al hospital por un cuadro de neumonía lobar inferior derecha, endocarditis bacteriana, insuficiencia renal crónica agudizada y trombocitopenia autoinmune. Durante su evolución, presentó hemorragia digestiva, la cual conllevó a choque hipovolémico en dos ocasiones. En la segunda colonoscopia, durante la retirada del colonoscopio, aproximadamente a $12 \mathrm{~cm}$ del margen anal, se encontró un vaso arterial aberrante con sangrado activo. Por ello, se realizó hemostasia con adrenalina diluida 1:10,000 y se colocaron dos endoclips con la resolución completa del sangrado. El paciente se recuperó del estado de choque y no presentó sangrado digestivo de nuevo, pero falleció a las dos semanas por una nueva neumonía y falla orgánica múltiple.

Palabras clave: Hemorragia digestiva, Dieulafoy, recto, endoscopia.

\section{INTRODUCCIÓN}

La hemorragia gastrointestinal es un trastorno frecuente, tanto en consulta externa como en la Sala de Urgencias, pudiendo ser causada por diversas etiologías. La lesión de Dieulafoy, definida como una anomalía vascular que se

\section{Abstract}

Gastrointestinal bleeding is a frequent disease in consultation and in the emergency room which has very different etiologies. Dieulafouy's lesion, described for the first time in 1898 is one of the rarest but potentially mortal lesion because of its diagnostic difficulty. We present the case of an 81 year old male patient who arrived to the hospital with a right inferior lobar pneumonia, bacterial endocarditis, acute chronic renal failure and autoimmune thrombocytopenia. During the hospital evolution the patient started with gastrointestinal bleeding that produced an hypovolemic shock twice, during the second colonoscopy in the colonoscope extraction approximately at 12 $\mathrm{cm}$ from the anal margin we found an aberrant arterial vessel with active bleeding. We performed hemostasis with diluted adrenalin 1:10,000 and with application of two endoclips with the complete resolution of the bleeding. The patient recovered from the hypovolemic shock with no signs of a new bleeding; nevertheless, he died two weeks after the bleeding event with a multiple organic failure secondary to another pneumonia.

Key words: Gastrointestinal bleeding, Dieulafoy, rectum, endoscopy.

presenta como una arteria tortuosa y de gran calibre, fue descrita por primera vez en el año de 1898. Esta lesión es una de las causas más raras, pero tiene gran relevancia por el riesgo de morbimortalidad que representa. De hecho, la lesión de Dieulafoy es una condición rara, pero potencialmente mortal.
* Servicio de Cirugía Gastrointestinal. Hospital Ángeles León, Guanajuato, México.

‡ Departamento de Medicina y Nutrición. Universidad de Guanajuato, México.

www.medigraphic.com/actamedica
Correspondencia:

Dr. Benjamín Gallo Arriaga

Correo electrónico: benjasgallo@gmail.com

Aceptado: 26-09-2019.

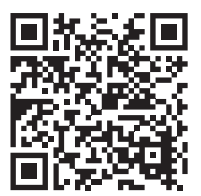




\section{REPORTE DEL CASO}

Se trata de un hombre de 81 años que ingresó a la Unidad de Cuidados Intensivos por presentar cuadro de neumonía lobar inferior derecha, endocarditis bacteriana, insuficiencia renal crónica agudizada y trombocitopenia autoinmune.

Entre sus antecedentes, estaban: portador de marcapaso bicameral por enfermedad del nodo sinoauricular $(\mathrm{SA})$, portador de filtro de vena cava inferior por evento trombótico pulmonar previo con sospecha de síndrome antifosfolípidos, así como manejo con acenocumarina durante seis años, el cual posteriormente sólo lo manejó con aspirina, además de portador de enfermedad diverticular de colon.

A su ingreso a la Unidad de Cuidados Intensivos, fue manejado con antibióticos de amplio espectro para la neumonía y endocarditis (se aisló Enterococcus fecalis), y manejo sustitutivo para la función renal.

Posteriormente, se valoró por Cirugía Cardiotorácica, quienes decidieron realizar ecocardiograma transesofágico, el cual reportó insuficiencia aórtica severa y dos vegetaciones grandes en dicha válvula. Debido a esto se decidió someter al paciente a un recambio valvular aórtico con prótesis biológica.

El paciente soportó la cirugía cardiaca, sin embargo, su evolución no fue del todo satisfactoria, cursando con trombocitopenia persistente y dependencia de ventilación mecánica asistida. A las dos semanas de la cirugía, y en cuidados intermedios, presentó cuadro severo de sangrado digestivo alto, por lo que se realizó panendoscopia, y se encontró pangastritis con hemorragia activa, la cual se controló con aplicación de argón-plasma.

A la semana después de este evento, presentó un cuadro de rectorragia masiva con choque hipovolémico. Se manejó con aminas, hemoderivados y soluciones, logrando

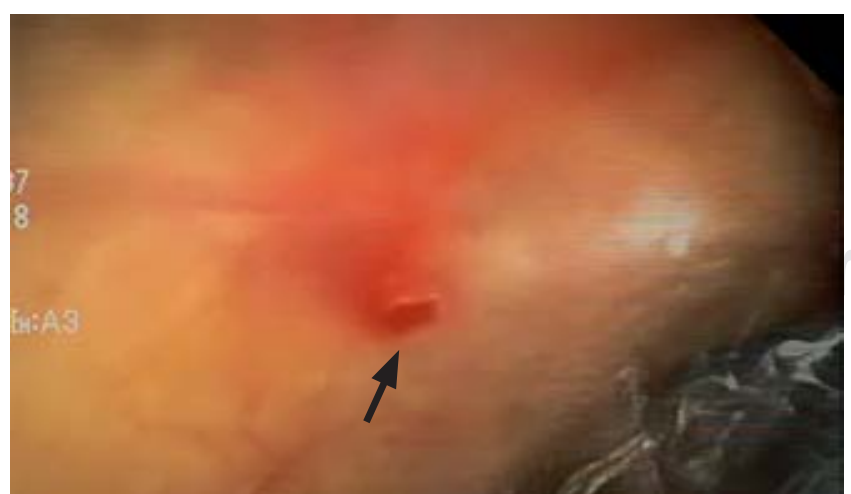

Figura 1: Lesión de Dieulafoy, previo lavado con agua (flecha negra).

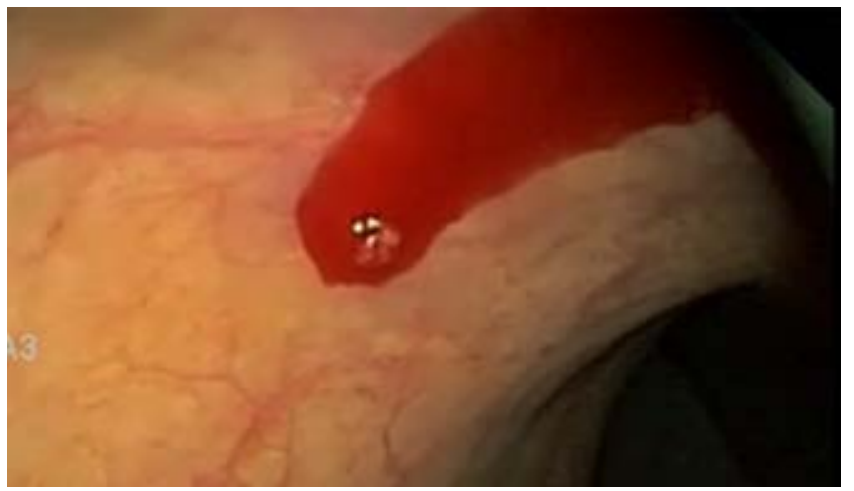

Figura 2: Lesión de Dieulafoy con sangrado activo.

estabilizarlo y disminuir considerablemente el sangrado. Sin embargo, a las cuatro horas, volvió a presentar rectorragia masiva, por lo que, de nuevo, se manejó el estado de choque. Una vez estabilizado, se realizó colonoscopia, y durante ésta, se valoró el sigmoides sin encontrar sitio de sangrado a nivel de los divertículos. Durante la retirada del colonoscopio, aproximadamente a $12 \mathrm{~cm}$ del margen anal, se encontró un vaso arterial aberrante con sangrado activo (Figuras 1 y 2), por lo que se procedió a realizar hemostasia con adrenalina diluida 1:10,000 (Figura 3) y se colocaron dos endoclips con la resolución completa del sangrado (Figura 4).

El paciente recuperó la estabilidad hemodinámica, y se transfundió hasta llegar a cifras de hemoglobina de $11 \mathrm{mg} /$ dL. Ya no volvió a presentar sangrado rectal y continuó su evolución en terapia intensiva. Finalmente, falleció a las dos semanas debido a una nueva neumonía, que ocasionó falla orgánica múltiple.

\section{DISCUSIÓN}

La lesión de Dieulafoy (LD) es una condición rara con un riesgo alto de mortalidad. Esta lesión consiste en una arteria dilatada y tortuosa ubicada en la mucosa, que puede resultar en un sangrado gastrointestinal significativo junto con sus complicaciones. ${ }^{1-3}$ Ésta constituye de 1 a $2 \%$ de todas las causas de sangrado digestivo, de las cuales $70 \%$ se encuentran en el estómago. El otro $30 \%$ se distribuye a lo largo de todo el tubo digestivo, siendo el duodeno y el colon los más afectados; menos de $2 \%$ del total de las LD se detectan en el recto. Este último tipo, es más prevalente en hombres en la secta y séptima década de la vida; su tasa de mortalidad varía de 8 a $17 \% .1,4,5$

Los factores de riesgo más significativos para una LD son: el uso de agentes antiplaquetarios $(p=0.022)$ y el alcohol $(p=0.001) .{ }^{5}$ Sin embargo, se ha descrito que la 
enfermedad renal crónica, hipertensión y quemaduras mayores podrían verse relacionados. ${ }^{2}$

Característicamente, las lesiones son arterias prominentes en la submucosa, con un calibre de 1 a $5 \mathrm{~mm}$, lo cual corresponde a 10 veces el tamaño de una arteria normal a ese nivel. ${ }^{3}$ La erosión de la capa mucosa y la pared arterial son las responsables del sangrado; éste suele ser intenso, pulsátil y lleva al paciente a presentar estado de choque. Esta lesión se distingue de las úlceras pépticas, ya que la fibrosis subintimal y la mucosa carecen de infiltrado inflamatorio. ${ }^{5}$

La patogénesis del sangrado por una LD todavía no es clara, aunque se incluye atrofía e isquemia de la mucosa, añadida a una presión ejercida por cada latido de la arteria dilatada, lo que lleva a la erosión del epitelio que recubre la mucosa. ${ }^{3}$ En lesiones ubicadas en el tubo digestivo bajo, se ha propuesto un mecanismo adicional, donde la materia fecal sólida contribuye a la erosión estercorácea sobre la anormalidad vascular. ${ }^{3}$

La localización rectal de la lesión de Dieulafoy se puede presentar como un sangrado abundantante o intermitente, sin necesidad de acompañarse de dolor. ${ }^{1,3,4}$ Gracias a los avances en la endoscopia terapéutica, la mortalidad de esta lesión ha disminuido drásticamente (de 80 a $8 \%$ ). ${ }^{3}$

Hasta el día de hoy, no hay aún un consenso para el tratamiento de la lesión de Dieulafoy. Las opciones de tratamiento dependen del modo de presentación, sitio de la lesión y la experiencia disponible. ${ }^{3}$ Entre las técnicas de diagnóstico y tratamiento, la endoscopia se sitúa como la herramienta de elección; su tasa de éxito varía de 75 a100\%, aunque se puede dificultar en la etapa inicial del sangrado por el tamaño pequeño de la lesión, el patrón intermitente y una mala preparación colónica. ${ }^{2,5,6}$ Esto provoca que, en ocasiones, se requiera hacer endoscopias repetidas. ${ }^{1}$ A pesar de que existen varios métodos terapéuticos endoscópicos utilizados para el tratamiento de la lesión de Dieulafoy, aún no está claro cuál es el mejor.

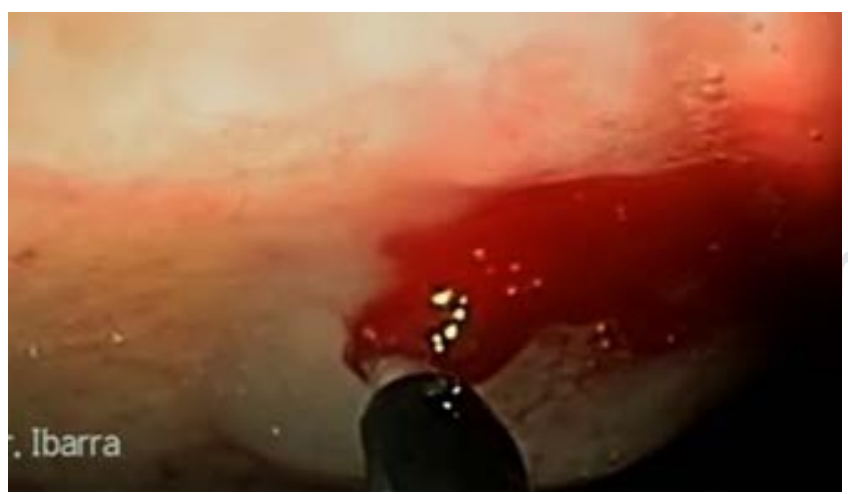

Figura 3: Inyección de adrenalina. Se observa la elevación de la mucosa.

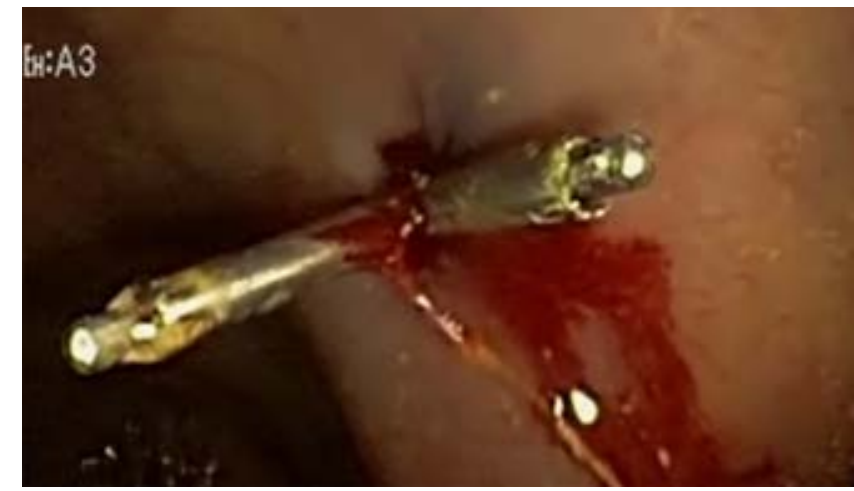

Figura 4: Endoclips colocados y cese del sangrado activo.

El tratamiento con clips hemostáticos ha demostrado en algunos artículos dar mejores resultados que la inyección endoscópica de epinefrina..$^{4,7}$

Sin embargo, Jeon y colaboradores ${ }^{3}$ reportaron que la combinación de dos métodos terapéuticos endoscópicos es muy superior a la monoterapia, ya que tiene una menor tasa de resangrado. Suele utilizarse un método mecánico (clips hemostáticos o ligaduras con banda) y la inyección de epinefrina en la submucosa. La angiografía y las intervenciones quirúrgicas actualmente sólo están justificadas si los métodos endoscópicos fallan. ${ }^{3}$

\section{CONCLUSIÓN}

En pacientes con sangrado del tubo digestivo bajo, sobre todo en sangrados agudos y profusos, y en aquellos pacientes con hemorragia masiva que no se acompaña de dolor, el cirujano debe tener en mente, como diagnóstico diferencial, la lesión de Dieulafoy. El abordaje debe realizarse primero con estabilización hemodinámica del paciente y después mediante abordaje endoscópico para la visualización directa de la lesión; asimismo, se debe realizar su manejo definitivo en el mismo procedimiento.

El uso de dos métodos endoscópicos para tratar el sangrado de este origen siempre será el tratamiento de elección. Este abordaje fue el que se utilizó en nuestro paciente, y con ello se logró detener el sangrado por completo.

\section{REFERENCIAS}

1. Khan R, Mahmad A, Gobrial M, Onwochei F, Shah K. The diagnostic dilemma of Dieulafoy's lesion. Gastroenterol Res. 2015; 8 (3-4): 201-206. Available from: http://www.ncbi.nlm.nih.gov/ pubmed/27785297

2. Nadhem ON, Salh OA, Bazzaz OH. Lower gastrointestinal bleeding due to rectal Dieulafoy's lesion. SAGE Open Med Case Reports. 2017; 5: 2050313X1774498.

3. Jeon HK, Kim GH. Endoscopic management of Dieulafoy's lesion. Clin Endosc. 2015; 48 (2): 112-120. 
4. Tursi A. Rectal Dieulafoy lesion. Clin Res Hepatol Gastroenterol. 2017; 41 (1): 1-2. Available from: http://www.ncbi.nlm.nih.gov/ pubmed/26186876

5. Shin HJ, Ju JS, Kim KD, Kim SW, Kang SH, Kang SH et al. Risk factors for Dieulafoy lesions in the upper gastrointestinal tract. Clin Endosc. 2015; 48 (3): 228-233.

6. Lara LF, Sreenarasimhaiah J, Tang S, Afonso BB, Rockey DC. Dieulafoy lesions of the Gl tract: localization and therapeutic outcomes. Dig
Dis Sci. 2010; 55 (12): 3436-3441. Available from: http://www.ncbi. nlm.nih.gov/pubmed/20848205

7. Chung I-K, Kim E-J, Lee M-S, Kim H-S, Park S-H, Lee M-H, et al. Bleeding Dieulafoy's lesions and the choice of endoscopic method: Comparing the hemostatic efficacy of mechanical and injection methods. Gastrointest Endosc. 2000; 52 (6): 721-724. Available from: https://www.sciencedirect.com/science/article/pii/ S0016510700701830 